\title{
0.35 Tesla magnetic resonance imaging findings in a cohort of 399 seizure patients. Experience from a single centre in Nigeria
}

\author{
Birinus A Ezeala-Adikaibe, ${ }^{1,2}$ Bibiana Oti, ${ }^{1,3}$ Samuel C. Ohaegbulam, ${ }^{1}$ Okwudili Okwuonodulu, ${ }^{1}$ \\ Chika Ndubuisi ${ }^{1,4}$ \\ ${ }^{1}$ Memfys Hospital for Neurosurgery Enugu; ${ }^{2}$ Neurology Unit Department of Medicine University of Nigeria \\ Teaching Hospital Enugu; ${ }^{3}$ Neurology Unit Department of Medicine, Enugu State Teaching Hospital, \\ Enugu; ${ }^{4}$ Neurosurgery Unit Department of Surgery, Enugu State Teaching Hospital, Enugu, Nigeria
}

\begin{abstract}
Epilepsy/seizures are major indications for brain imaging in clinical neurology. Structural lesions that may cause seizures are numerous and are defined using various neuroimaging techniques, including magnetic resonance imaging. The resolution of MRI allows for better fine ultra-structural lesions delineation. The aim of this study was to describe the pattern and frequency of structural
\end{abstract}

\begin{abstract}
Correspondence: Birinus A. Ezeala-Adikaibe, Neurology Unit, Department of Medicine University of Nigeria Teaching Hospital Enugu, P.M.B 01129, Enugu, Nigeria. Tel.: 08036769469.

E-mail: birinus.ezeala-adikaibe@unn.edu.ng
\end{abstract}

Key words: Epilepsy; seizures; epileptogenic lesions; MRI; Nigeria.

Acknowledgment: The authors wish to acknowledge the help and support of the secretarial staff of Memfys Hospital.

Conflict of interest: The authors declare no conflict of interest.

Availability of data and materials: All data generated or analyzed during this study are included in this published article.

Ethics approval and consent to participate: The Ethics Committee of Memfys Hospital for Neurosurgery approved this study (APPROVAL CODE : MHN/BO/BEA/SCO/20). The study is conformed with the Helsinki Declaration of 1964, as revised in 2013, concerning human and animal rights. All patients participating in this study signed a written informed consent form for participating in this study.

Informed consent: Written informed consent was obtained from a legally authorized representative(s) for anonymized patient information to be published in this article. [This was a retrospective study hence written informed consent was not necessary or available as to personal information was disclosed].

Received for publication: 27 December 2021.

Revision received: 7 February 2022.

Accepted for publication: 7 February 2022.

This work is licensed under a Creative Commons Attribution NonCommercial 4.0 License (CC BY-NC 4.0).

(C) Copyright: the Author(s), 2022

Licensee PAGEPress, Italy

Annals of Clinical and Biomedical Research 2022; 3:188

doi:10.4081/acbr.2022.188 brain lesions in MRI of patients with seizures and no clinically evident focal neurological signs. This was a retrospective, descriptive study carried out in a private hospital in Enugu, South East Nigeria to review all MRI results of patients who presented with seizures without clinical evidence of focal neurologic deficits. The MRI reports of two-third of the patients $(47.9 \%)$ revealed focal lesions and about a third of the patients (32.2\%) had normal findings. The structural lesions reported were mostly brain tumors (16\%), stroke (9.5\%), central nervous system infections $(6.5 \%)$, brain malformation $(6 \%)$ and encephalomalacia/gliosis (5\%). Frequency of focal lesions clearly increased with age. Young patients were mostly associated with normal findings. Brain tumors and stroke were noted to occur more in the middle and aged patients respectively. Brain Magnetic Resonance Imaging remains a useful tool in the workup of patients with seizures without neurologic deficits. Treatable lesions can easily be revealed using this imaging modality.

\section{Introduction}

Epilepsy is one of the major indications for brain imaging in clinical neurology. ${ }^{1}$ The dramatic nature of epileptic seizures, especially new-onset seizures in adults, may compel patients and their families to insist on neuroimaging as well as other relatively expensive investigations without recourse to guidelines. Structural lesions that may cause seizures are numerous usually defined using various neuroimaging techniques, including Magnetic Resonance Imaging (MRI). Structural MRI findings in seizures/epilepsy are similar to those seen in Computerized Tomography (CT), however, because of better resolution of MRI, ultra-structural lesions are better delineated depending on the resolution of the MRI and imaging protocol used. ${ }^{1,2}$ Structural changes on MRI in seizures can be categorized into the following groups: mesial temporal sclerosis, cortical developmental malformations/neuronal migration disorders, phacomatoses, vascular abnormalities, infections, neoplasms, stroke, traumatic lesions and miscellaneous conditions such as gliosis, encephalomalacia and encephalocele. ${ }^{1-6}$ A good proportion of these disorders are amenable to surgical treatment, hence the need for proper imaging. ${ }^{4-6} \mathrm{MRI}$ is also useful in the following up of patients on anti-epileptic drugs such as Vigabatration ${ }^{7}$ while negative or normal MRI findings are also important in reassuring patients, thus encouraging them to continue on conservative treatment where necessary.

The pattern of structural MRI lesions or other radiologic imaging findings in epilepsy depend on the age group studied and this may generally reflect the epidemiology of diseases in the region studied. ${ }^{5,810}$ For example, in Sub Saharan Africa, lesions resulting from infections and trauma may be significant. Other factors that may affect MRI findings in seizure in our setting include the avail- 
ability and expertise of the neuro radiologist, and the spatial resolution of the MRI machine.

The International League Against Epilepsy (ILAE) suggests that everyone with epilepsy should have, in the ideal situation, a high-quality MRI. ${ }^{11}$ The National Institute of Health and Clinical Excellence guidelines recommend that MRI of the brain should be the investigation of choice in children and adults with epilepsy to screen for structural abnormalities. ${ }^{12}$ In CT based studies, the frequency of structural lesions ranged from $6 \%$ to $55.1 \%$ depending on methodology employed. ${ }^{8-10}$ The three commonest structural findings in the CT of people with epilepsy reported in Enugu were tumors (20.4\%), encephalomalacia (18.9\%) and strokes $(7.7 \%) .{ }^{8-10}$ The yield of MRI also varies depending on the cohort studied: new onset seizures, epilepsy patients or intractable seizures. . $^{8-11,13,14}$ Epileptogenic lesions were detected in $12.7-14 \%$ in patients with first - ever seizures ${ }^{15,16}$ and in up to $82-86 \%$ in people with intractable seizures. ${ }^{17}$ The presence of MRI abnormalities in patients with new-onset epilepsy is predictive of seizure recurrence and difficulty with seizure control using medical therapy. ${ }^{17}$

In Nigeria, MRI is gradually replacing CT in the evaluation and management of patients with seizures and epilepsy. The use of standard epilepsy protocol in the evaluation of patients with seizures is recommended, ${ }^{11}$ Unfortunately, in most settings in Nigeria, a non-epilepsy protocol MRI is usually performed primarily because of cost and lack of specialized neuro radiologists. Structural lesions are more frequently found using epilepsy protocols compared to non-epilepsy protocols. ${ }^{19}$

Review of the pattern of structural lesions in brain MRI of seizure patients is important in the determination of causes of seizures and epilepsy in tropical region, where such data have not been documented.

The aim of this study was to review the pattern of focal lesions in patients presenting with seizures not presenting with focal neurological deficits in a tertiary hospital in South East Nigeria.

\section{Materials and Methods}

This was a retrospective, descriptive study of brain MRI of patients seen in a private tertiary hospital in Enugu, the capital of Enugu State, South East Nigeria. The hospital is a referral neurology/neurosurgery center and introduced the first MRI scan in
South East Nigeria and receives from all parts of Nigeria. At the time of the study $0.35 \mathrm{~T}$ MRI was the only MRI machine available in the hospital. Although most of the studied patients scanned were referred from other hospitals however, a good number of them were primary patients of the hospital.

Brain MRI was performed at various intervals from the onset of ailment. Data on age, gender, symptoms and radiologic findings were entered into a questionnaire and transferred into personal computer. Routine non-contrast and contrast brain MRI were conducted using BT1-0.35T system. Images were obtained in sagittal T1, T2, GRE, FLAIR; axial and coronal T1, T2 sequences. We reviewed only those with the diagnosis of seizure and/ or epilepsy. MRI was reported by consultant radiologists and reviewed by two neurologists. MRI reports included in the study were done between December 2010 and February 2018.

\section{Inclusion and exclusion criteria}

All adult cases (18 years and above) with a diagnosis of or suspected seizures were included if the meet the study criteria. Patients with documented clinical signs suggestive of acute or subacute focal neurologic deficits were excluded. History of brain surgery was also excluded. Other cases that were excluded were those with metabolic disorders such as major organ dysfunction, diabetic coma and cases of possible mental illness especially if indicated in the request notes. In the case of repeat MRI, the last scan was selected.

Table 1. Age and gender distribution of the Patients.

\begin{tabular}{lcccc} 
Gender & Male & Female & Total & p-value \\
20 & $12(5)$ & $10(6.3)$ & $22(5.5)$ & \\
$20-34$ & $88(36.5)$ & $75(47.5)$ & $163(40.9)$ & \\
\hline $34-44$ & $31(12.9)$ & $25(15.8)$ & $56(14)$ & \\
$45-54$ & $31(12.9)$ & $8(5.1)$ & $39(9.8)$ & \\
\hline $55-64$ & $30(12.4)$ & $10(6.3)$ & $40(10)$ & \\
$\geq 65$ & $49(20.3)$ & $30(19)$ & $79(19.8)$ & 0.02 \\
\hline Mean age (sd) & $43.6 \pm 19.1$ & $40.1 \pm 19.3$ & $42.2 \pm 19.2$ & 0.07 \\
Median age & 41 & 33 & 37 & \\
\hline Total & $241(60.4)$ & $158(39.6)$ & $399(100)$ & \\
\hline \multicolumn{5}{c}{}
\end{tabular}

Table 2. Distribution of MRI findings.

\begin{tabular}{|c|c|c|c|c|}
\hline Normal MRI & \multicolumn{2}{|c|}{$\begin{array}{l}\text { Epileptogenic lesions } \mathrm{N}=191(47.9) \\
\text { (focal lesions with strong epileptogenic potential) }\end{array}$} & \multicolumn{2}{|c|}{$\begin{array}{l}\text { Possibly related to causes of epilepsy } \\
\text { (non specific findings) }\end{array}$} \\
\hline & Tumors & $64(16)$ & Brain atrophy & $45(11.3)$ \\
\hline & Stroke & $38(9.5)$ & DWM$^{*}$ & $31(7.8)$ \\
\hline & Infections & $26(6.5)$ & Hydrocephalus & $3(0.8)$ \\
\hline & Congenital abnormalities & $26(6.5)$ & $\mathrm{HWML}^{* *}$ & $1(0.3)$ \\
\hline & Encephalomalacia/gliosis & $20(5)$ & & \\
\hline & Cysts & $8(2)$ & & \\
\hline & Subdural hematoma & $2(0.5)$ & & \\
\hline & Inflammatory & $2(0.5)$ & & \\
\hline & Contusion & $1(0.3)$ & & \\
\hline & Hyperostosis frontalis & $1(0.3)$ & & \\
\hline & Calcification & $1(0.3)$ & & \\
\hline & Mesial temporal lobe sclerosis & $1(0.3)$ & & \\
\hline Total*** & $128(32.1)$ & $191(47.9)$ & & $80(20.1)$ \\
\hline
\end{tabular}

*Deep white matter lesions. ${ }^{* *}$ Hyperintense white matter lesions. ${ }^{* * *}$ Percentage of all scans reviewed 


\section{Ethics approval}

The study was reviewed and approved by the hospital's Ethics Committee.

\section{Statistical Methods}

For database management and statistical analyses, we used the SPSS version 23 (IBM Corporation, New York, USA). Data were presented in tables and figures. Continuous variables, mean values and standard deviation were calculated. Rates were expressed as percentages. Categorical values were compared using the Chi Square test. Mean age was compared using independent t-test. In all, $p$ value $<0.05$ was regarded as statistically significant. Conclusions were drawn at $95 \%$ confidence interval.

\section{Results}

A total of 399 MRI reports were reviewed and $60.4 \%$ were males and the rest females. Most of the participants were aged 2034 years. $\mathrm{P}=0.02$ The mean and median ages of the patients were $42.2 \pm 19.2$ and 37 years respectively (Table 1). The MRI report of most patients $(\mathrm{n}=191,47.9 \%)$ revealed focal lesions. While that of $80(20.1 \%)$ revealed lesions, which may share common risk factors with epilepsy, hence may indirectly suggest secondary seizures. About a third of the patients had normal MRI 128 (32.1\%).

The commonest structural lesions reported were brain tumors $64(16 \%)$, stroke $38(9.5 \%)$, central nervous system infections 26 $(6.5 \%)$, brain malformation $24(6 \%)$ and encephalomalacia/gliosis $20(5 \%)$. Other structural findings are listed on Tables 2 and 3. Lesions such as grey matter heterotropia, multiple sclerosis, white matter hyperintensities and mesial temporal sclerosis were few but clearly documented.

The age distribution of epileptogenic lesions clearly increased with age reaching as high as $57.5 \%$ in the 55-64 age group while that of indirect indicators of secondary seizures peaked from 65 years and above. About $44.7 \%$ of patients $20-44$ years had normal MRI compared to $7.6 \%$ of patients 65 years and above (Figure 1). The age distribution of the major lesions is shown in Figure 2. Brain tumors peaked at 55-64 years while stroke peaked after 65 years. The mean age of diagnosis of various lesions are shown in Table 4. Stroke increased steadily from 20 years peaking at 65 years and above. Tumors peaked at 55-64 years and infections at 45-54 years. Brain malformations were more frequent before 20 years of age. The age of diagnosis of encephalomalacia/gliosis and brain malformations did not significantly differ from the age of

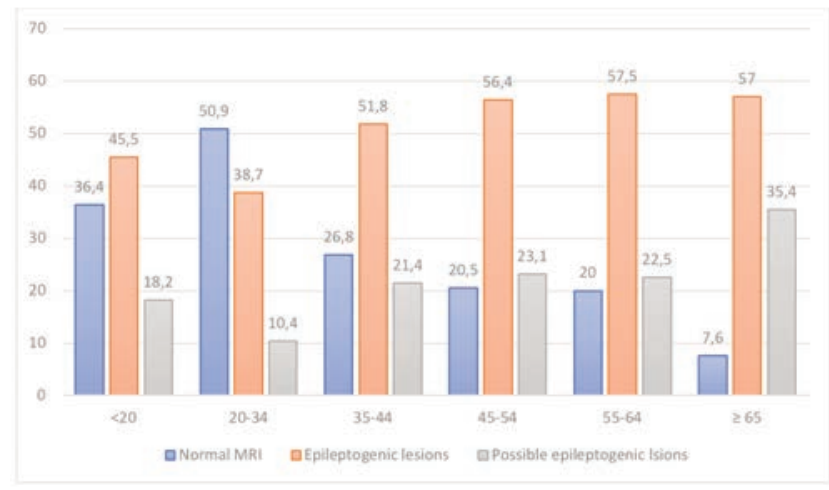

****Possible epileptogenic lesion

Figure 1. Distribution of lesion epileptogenic, possible epileptogenic and Normal MRI scans. patients with normal MRI (Table 3).

\section{Discussion}

The fundamental goal of seizure workup is to determine, if possible, any underlying structural etiology. Brain MRI provides

Table 3. Distribution of epileptogenic lesions.

\begin{tabular}{lcc}
\hline Epileptogenic lesions $\mathrm{N}=191(\mathbf{4 7 . 9}) *$ & $\mathbf{N}(\%) * *$ & Sub total \\
Stroke & & \\
- Infarct & $36(94.7)$ & \\
- Hemorrhage & $2(5.3)$ & 38 \\
Encephalomalacia & $18(90)$ & \\
Gliosis & $2(10)$ & 20 \\
Infective & & \\
- Encephalitis & $13(50)$ & \\
- Cerebritis & $5(19.3)$ & \\
- Cerebral abscess & $3(11.5)$ & \\
- Meningitis & $2(7.7)$ & \\
- Granulomatous necrosis & $2(7.7)$ & \\
- Toxoplasmosis & $1(3.8)$ & 26 \\
Congenital & $12(46.2)$ & \\
- AVM* & $5(19.3)$ & \\
- Brain malformation & $4(15,4)$ & \\
- Dandy Walker syndrome & $1(3.8)$ & \\
- Mucocele & $1(3.8)$ & \\
- Agenesis of corpus callosum & $1(3.8)$ & \\
- Grey matter heterotropia & $1(3.8)$ & 26 \\
- Ventriculomegaly &
\end{tabular}

*Percentage of all scans reviewed. **Percentage of all subtotal.

Table 4. Age and gender distribution of the Patients.

\begin{tabular}{lcc} 
Gender & Mean age (sd) & p-value* \\
Normal MRI & $32.4(14.2)$ & - \\
Brain Tumors & $45.8(16.1)$ & $<0.01$ \\
\hline Stroke & $62.7(15.6)$ & $<0.01$ \\
CNS infections & $45.2(20.7)$ & $<0.01$ \\
\hline Encephalomalacia/Gliosis & $39.1(20.3)$ & 0.47 \\
Brain Malformations & $27.2( \pm 11.9)$ & 0.65
\end{tabular}

*In comparison to mean age of patients with Normal MRI.

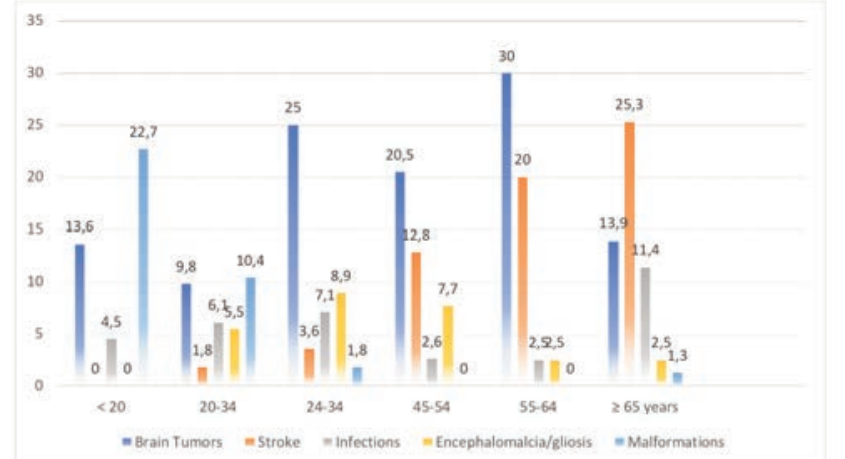

Figure 2. Distribution of most common epileptogenic lesions. 
valuable information in this regard, hence has implications in treatment selection and prognosis. This is of a special importance in new-onset adult seizures as well inpatients with medically intractable seizures. The increasing availability of MRI in Nigeria has significantly improved diagnosis and successful surgical treatment of epilepsy patients with resectable focal lesions.

Overall, this study suggests that a large proportion of patients with seizures may have focal lesions based on the high rates of epileptogenic and possible epileptogenic lesions reported. These findings also offer an insight into the causes and risk factors for epilepsy in Nigeria. Previous studies on structural brain findings in seizures/epilepsy in Nigeria were based on CT imaging. ${ }^{8-10}$ Oguniyi et al. ${ }^{9}$ reported a frequency of $45.3 \%$ of abnormal CT scans while a similar study at Enugu reported a diagnostic yield of $55.1 \% .^{8}$

The commonest structural lesions reported were brain tumours $64(16 \%)$ and stroke $38(9.5 \%)$. MRI sensitive lesions such as grey matter heterotropia, multiple sclerosis, white matter hyperintensities and mesial temporal sclerosis were few but clearly documented. Brain tumors peaked at 55-64 years while stroke peaked after 65 years.

The mean age and age distribution of patients in this study is different from the age distribution of epilepsy cohorts reported from several studies in Nigeria. ${ }^{20,21}$ The reasons are not unrelated to the inclusion of both patients with potential epilepsy and those with symptomatic seizures. The distribution of epileptogenic lesions varied with age. The mean age of patients with tumors was 45.8 (16.1) years and the mean age of patients with stroke was 62.7 (15.6) years. We have reported an increasing diagnostic yield of neuro imaging in people with epilepsy using brain CT especially after 59 years. In this study, frequency of epileptogenic lesion almost leveled out after 44 years probably due to better resolution of MRI when compared to CT.

Similar to other studies on structural findings in epilepsy, ${ }^{13-15}$ tumors were common significant neurological findings in this study. Although about $4 \%$ of epilepsy patients may develop brain tumors over their lifetime, incidence of seizures in patients with brain tumors may be as high as $30 \%$ to $100 \%$ depending on tumor type and location. ${ }^{17}$ Low grade astrocytoma and gliomas, cortical location and temporal lobe tumors are reported to be highly epileptogenic. ${ }^{22,23}$ We are not aware of any study that has evaluated pattern of MRI findings in people with epilepsy in Nigeria. Most of other local studies used brain CT imaging technique. For instance, in a CT based study in adolescent seizure patients, ${ }^{13}$ tumors were reported in $27.3 \%$ in Saudi Arabia ${ }^{24}$ and in 20.4\% Enugu. ${ }^{8}$ Comparing with these CT studies, the proportion of tumors in the index is lower; however, this may only be relative. This observation could be due to: i) inclusion of more elderly people with higher rates of stroke and brain atrophy, ii) inclusion of both epilepsy and new-onset seizures, and iii) the overall higher sensitivity of MRI in detecting finer and subtle structural changes than CT.Furthermore, CT might have been used as a first line investigative modality before MRI.

Stroke was diagnosed in $38(9.5 \%)$ of the patients in the index study. The rising incidence of seizures/epilepsy in the elderly has been attributed to several factors including stroke. ${ }^{25}$ Stroke is the leading cause of epilepsy in older adults and a leading cause of status epilepticus. ${ }^{25}$ In the developed countries, post stroke epilepsy accounts for $14 \%$ to $21 \%$ of all cases of epilepsy. ${ }^{26}$ Current demographic projections indicate a rising proportion of older adults in Nigeria $^{27}$ the population at greatest risk of stroke. Stroke type, the location of the lesion and family history of epilepsy are important determinants of post stroke seizures. ${ }^{28-29}$
Overall, seizures occur in about 3\%-6.4\% of ischemic stroke survivors within a year and $54 \%-66 \%$ of them go on to develop epilepsy. ${ }^{30-32-35}$ With the current prevalence of $12 / 1000,{ }^{33}$ the burden of post stroke epilepsy is set to increase in the community because the long-term cumulative risk of post-stroke epilepsy is also high. This study also gives some insight into this emerging problem. The mean age of patients with stroke in this study was 62.7 years similar to the age of stroke survivors in Nigeria. ${ }^{33}$ Other reasons may not be unrelated to the inclusion of both patients hydrocephalus, sub-dural hematoma and dementia which are possible causes of symptomatic seizures and epilepsy. The high rates of stroke in this study demonstrated the growing burden of post stroke epilepsy. The prevention of epilepsy is eminently illustrated in the case of post stroke epilepsy because of the several modifiable risk factors associated with stroke. The current study lends an impetus not only to the primary prevention of stroke but also of epilepsy.

The pattern of CNS infections in the current study was dominated by encephalitis however all the spectrum of CNS infections seemed to be represented. Considering the epidemiology of these disorders, it is possible that the current finding will be a gross underestimate of the contribution of CNS infections to seizures and epilepsy in our region. This is because most people at risk of CNS infections may not afford MRI at current costs. The study did not report any cysticercosis which has been reported elsewhere. ${ }^{34}$

Encephalomalacia, gliosis and brain atrophy add up to about $16 \%$ of all cases with positive MRI findings. They form a common pathogenic pathway in the evolution of several CNS insults, including stroke, infections and trauma and have been associated with seizures and epilepsy. ${ }^{29}$ Persisting seizures in these lesions may be indirect indicators of chronicity. In patients with dementia, which is also risk factor for epilepsy, generalized atrophy is a common finding. Encephalomalacia and gliosis peaked at a later age than infectious diseases but earlier than stroke suggesting multiplicity of causes. CT studies have shown that encephalomalacia can occur in as high as $3.7-18.9 \%$ of patients with epilepsy. ${ }^{8-10}$

Arteriovenous malformation is the commonest brain malformation listed in the current study and it has been reported in about $5-6.5 \%$ of people with epilepsy in the general population. ${ }^{35}$ Intracranial vascular malformations, including arteriovenous malformations, confer a significant risk of first ever seizure and epilepsy. ${ }^{36-37}$ Location and type of lesion are strongly correlated with the epileptogenicity of the lesion. ${ }^{38}$ As expected, the frequency of malformations peaked below 20 years and sharply dropped by the age of 44 years. The small peak of malformations from 65 years may be due to aneurysms, which are known to enlarge with age. All other brain malformations reported in this study have been associated with epilepsy. ${ }^{39}$ Detecting brain malformations are some of the advantages of using MRI over CT. Although this study used a $0.35 \mathrm{~T}$ MRI, one case of grey matter heterotropia was detected. Other findings such as multiple sclerosis, mesial temporal lobe sclerosis and hyperintense white matter lesions also underscore the importance of MRI in the evaluation of patients in our setting.

Two other findings that merit commentary in this study are white matter lesions and hydrocephalus. White matter lesions are seen very frequently in the elderly and can co-occur in the same patient with any of the other lesions, especially stroke. The relationship between white matter lesions and epilepsy has not been well defined but may follow the same pathway as stroke considering that white matter lesions and stroke appear to share similar pathogenetic mechanisms. Furthermore, white matter lesions are frequently described in degenerative dementia, which in turn, are risk factors for epilepsy. Hydrocephalus is a complication of many 
CNS diseases, which can also cause epilepsy. Conditions, like meningitis, may occur many years before the development of hydrocephalus. Hydrocephalus therefore in the context of relevant medical history may be a pointer to secondary seizures/epilepsy. In a previous study conducted in the same centre using a CT hydrocephalus was reported in about $9.2 \%$ of the cases. ${ }^{8}$

$\mathrm{SDH}$ was found only in two cases $(0.5 \%)$ while contusion was seen in $1(0.3 \%)$. While these two conditions are recognized risk factor for posttraumatic seizures and epilepsy, ${ }^{40-42}$ they were excluded from the study as primary causes of epilepsy.

\section{Limitations}

We acknowledge some limitations in this study. Firstly, low resolution of the MRI machine (0.35T) used in this study used may not be ideal in evaluating a wide range of s of epilepsy etiologies, nevertheless it is still useful in delineating gross potentially epileptogenic lesions such as tumors. Secondly, focal lesions are not necessarily epileptogenic, even though the odd of having epilepsy with them is high some focal lesions may be completely innocent. Functional imaging techniques (SPECT, PET, fMRI, MEG -magnetoencephalography, EEGs) are more likely to determine which lesions are truly epileptogenic.

Again, the prohibitive cost of MRI investigation in our environment might have introduced selections bias, thus our study findings may not be generalizable to the entire population. Despite all these limitations, this study offers an insight into the pattern of possible epileptogenic lesions in the region.

\section{Conclusions}

MRI is a useful tool in the work-up of patients with seizures without neurologic deficits. It remains a useful tool to rule out treatable lesion in such patients in resource poor setting in spite of cost.

\section{References}

1. Gupta V, Bronen R A. MRI: Overview of MR Techniques for Epilepsy. In Chugani $\mathrm{H}$ eds. Neuroimaging in Epilepsy. University Press Scholarship Online Oxford Scholarship Online; 2011. doi: 10.1093/acprof:oso/ 9780195342765.001.000

2. Cendes F, Theodore W H, Brinkmann B H, et al. Neuroimaging of epilepsy Handb Clin Neurol 2016;136:9851014.

3. Whelan CD, Altmann A, Bot1 JA, et al. Structural brain abnormalities in the common epilepsies assessed in a worldwide ENIGMA study. Brain. 2018;141:391-408.

4. Prado J A-L. Structural magnetic resonance imaging in epilepsy. Radiología 2012;54:9-20.

5. Bano S, Yadav SN, Chaudhary V, Garga UC. Neuroimaging in epilepsy. J PediatrNeurosci 2011;6:19-26.

6. Zhao X, Zhou Z, Zhu W, Xiang H. Role of conventional magnetic resonance imaging in the screening of epilepsy with structural abnormalities: a pictorial essay Am J Nucl Med Mol Imaging 2017;7:126-37.

7. Wheless J W, Carmant L, Bebin M, et al. Magnetic resonance imaging abnormalities associated with vigabatrin in patients with epilepsy. Epilepsia 2009;50:195-205.

8. Ezeala-Adikaibe AB, Ohaegbulam SC, Ndubuisi CA. The pat- tern of significant lesions found in computerized tomography scan of recurrent seizure patients at a center in Enugu, Nigeria. Niger J Clin Pract 2017;20:1289-93.

9. Ogunniyi A, Adeyinka A, Fagbemi SO, et al. Computerized tomographic findings in adolescent and adult Nigerian epileptics. West Afr J Med 1994;13:128-31.

10. Ndubuisi CA, Mezue WC, Ohaegbulam SC, et al. Neuroimaging findings in pediatric patients with seizure from an institution in Enugu. Niger J Clin Pract 2016;19:121-7.

11. Bernasconi A, Cendes F, Theodore WH, et al. Recommendations for the use of structural magnetic resonance imaging in the care of patients with epilepsy: A consensus report from the International League Against Epilepsy Neuroimaging Task Force. Epilepsia 2019;60:1054-1068.

12. NICE. Epilepsy in adults Quality standard Published: 28 February 2013. Available from: www.nice.org.uk/ guidance/qs26

13. Longe AC, Omojola MF. Computed tomographic brain scan findings in Saudi epileptic patients. East Afr Med J 1994;71:567-70.

14. Mir S, Dr. Ahmad M, Dr. Kumari R. Assessment of CT scans findings in patients with seizures: An Imaging perspective. Sch J App Med Sci 2016;4:3909-15.

15. King MA, Newton MR, Jackson GD, et al. Epileptology of the first-seizure presentation: a clinical, electroencephalographic, and magnetic resonance imaging study of 300 consecutive patients. Lancet 1998:352:1007-11.

16. Berg AT, Testa FM, Levy SR, Shinnar S. Neuroimaging in children with newly diagnosed epilepsy: A community-based study. Pediatrics 2000;106:527-32.

17. Passaro EA. Neuroimaging in Epilepsy. Available from: https://emedicine.medscape.com/article/1155295

18. Von Oertzen J, Urbach H, Jungbluth S, et al. Standard magnetic resonance imaging is inadequate for patients with refractory focal epilepsy. J Neurol Neurosurg Psych 2002;73:643-7.

19. Wieshmann UC. Clinical application of neuroimaging in epilepsy. J Neurol Neurosurg Psych 2003;74:466-70.

20. Ezeala-Adikaibe BA, Orjioke C, Ekenze O, et al. Prevalence of active convulsive epilepsy in an urban slum in Enugu South East Nigeria. Seizure 2016;35:100-105.

21. Onwuekwe IO, Onodugo OD, Ezeala-Adikaibe B, et al. Pattern and presentation of epilepsy in Nigerian Africans: A study of trends in the southeast. Trans R Soc Trop Med Hyg 2009;103:785-9.

22. Englot DJ, Chang EC, Vecht CJ. Epilepsy and brain tumors. Handb Clin Neurol 2016;134:267-85.

23. de Groot M, Reijneveld JC, Aronica E, Heimans JJ. Epilepsy in patients with a brain tumour: focal epilepsy requires focused treatment Brain 2012:135;1002-16.

24. Obajimi MO, Fatunde OJ, Ogunseyinde AO, et al. Computed tomography and childhood seizure disorder in Ibadan. West Afr J Med 2004;23:167-72.

25. WHO. Epilepsy: a public health imperative. World Health Organization; 2019

26. Forsgren L, Beghi E, Oun A, Sillanpaa M. The epidemiology of epilepsy in Europe: a systematic review. Eur J Neurol 2005; 12:245-53.

27. Federal Republic of Nigeria 2006 Population and Housing Census. Population Distribution by Age and Sex. State and Local Government Area. National Population Commission Abuja: Nigeria, 2010.

28. Leone MA, Tonini MC, Bogliun G,Gionco M, Tassinari T, Bottacchi E, et al. On behalf of ARES (Alcohol Related 
Seizures) Study Group. Risk factors for a first epileptic seizure after stroke: a case control study. J Neurol Sci 2009; 277:138142

29. Ferlazzo E, Gasparini S, Beghi E, Sueri C, Ruso E, Leo A et al. On behalf of the Epilepsy Study Group of the Italian Neurological Society. Epilepsy in cerebrovascular diseases: review of experimental and clinical data with meta-analysis of risk factors. Epilepsia 2016;57:1205-14.

30. Eriksson H, Wirdefeldt K, Åsberg S, Zelano J. Family history increases the risk of late seizures after stroke. Neurology 2019;93:e1964-70.

31. Graham NSN, Crichton S, Koutroumanidis M, et al. Incidence and associations of poststroke epilepsy the prospective south london stroke register. Stroke 2013;44:605-11.

32. Graham NS, Crichton S, Koutroumanidis M, et al. Incidence and associations of poststroke epilepsy: the prospective South London Stroke Register. Stroke 2013;44:605-11.

33. Ekenze OS, Adikaibe EAB, Obinna O, et al. The prevalence of stroke survivors in urban slums in Enugu, Nigeria. Open J Prev Med 2019;9:51-67.

34. Millogo A, Nitiema P, Carabin H, et al. Prevalence of neurocysticercosis among people with epilepsy in rural areas of Burkina Faso. Epilepsia 2012;53:2194-202.

35. Parkinson D, Bachers G. Arteriovenous malformations. J
Neurosurg 1980;53:285-99.

36. Turjman F, Massoud TF, Sayre JW, et al. Epilepsy associated with cerebral arteriovenous malformations: a multivariate analysis of angioarchitectural characteristics. Am J Neuroradiol 1995; 16:345-50.

37. Moriarity JL, Wetzel M, Clatterbuck RE, et al. The natural history of cavernous malformations: a prospective study of 68 patients. Neurosurgery 1999;44:1166 -71.

38. Josephson CB, Leach JP, Duncan R, et al. Seizure risk from cavernous or arteriovenous malformations Prospective population-based study. On behalf of the Scottish Audit of Intracranial Vascular Malformations (SAIVMs) steering committee and collaborators. Neurology 2011;76;1548-54.

39. Unterberger I, Bauer R, Walser G W, Bauer G. Corpus callosum and epilepsies. Seizure 2016;37:55-60.

40. Crawford PM, West CR, Chadwick DW, Shaw MD. Arteriovenous malformations of the brain: natural history in unoperated patients. J Neurol Neurosurg Psychiatry 1986;49: $1-10$.

41. Frey L C. Epidemiology of Posttraumatic Epilepsy: A Critical Review. Epilepsia 2003;44:11-7.

42. Temkin N R. Risk Factors for Posttraumatic Seizures in Adults. Epilepsia 2003;44:18-20. 